\title{
Research of Dielectric Breakdown Microfluidic Sampling Chip
}

\author{
Feng Jiang, Yunwen Lei, and Jun Yu \\ School of Optical and Electronic Information, Huazhong University of Science and Technology, Wuhan, Hubei 430074, China \\ Correspondence should be addressed to Jun Yu; 635286773@qq.com
}

Received 27 January 2013; Accepted 1 February 2013

Academic Editor: Yongfeng Luo

Copyright ( 2013 Feng Jiang et al. This is an open access article distributed under the Creative Commons Attribution License, which permits unrestricted use, distribution, and reproduction in any medium, provided the original work is properly cited.

\begin{abstract}
Microfluidic chip is mainly driven electrically by external electrode and array electrode, but there are certain disadvantages in both of ways, which affect the promotion and application of microfluidic technology. This paper discusses a scheme that uses the conductive solution in a microchannel made by PDMS, replacing electrodes and the way of dielectric breakdown to achieve microfluidic chip driver. It could reduce the driving voltage and simplify the chip production process. To prove the feasibility of this method, we produced a microfluidic chip used in PDMS material with the lithography technology and experimented it. The results showed that using the dielectric breakdown to achieve microfluidic chip driver is feasible, and it has certain application prospect.
\end{abstract}

\section{Introduction}

Microfluidic chip analysis system is mainly using the manipulation of the fluid to finish the functions including sampling, dilution, plus sampling, reaction, separation, analysis, and detection in microchannel chip system [1]. Microfluidics have many types of driving and controlling technologies; the principle and form are different, which can be divided into pressure-driven, electrically driven, heat-driven, the surface tension-driven, centrifugal force drivers, and so forth $[2,3]$.

Electric driver is still one of the most commonly used and most effective drivers in microfluidic chip analysis systems. It usually places the outer electrode at both ends of the sump; by applying a voltage on the electrode, the driving electric field was formed in the solution to achieve the driving of the liquid in the microchannel. Currently, this is the mainstream drive mode of the chip electrophoresis analysis system.

Usually, electrically driven driver has two ways. One is the chip external electrode way, such as those chips which designed by Harrison et al. [4], Schasfoort et al. [5], Bianchi et al. [6], Locascio et al. [7], Pittman et al. [8], and Sun et al. [9]. The other way is arraying electrode on chip, such as those designed by Lin and $\mathrm{Wu}[10,11]$ and Fu and Yang [12]. Both ways, however, have some defects. The former requires a high-voltage power supply; there are security and large volume problems; the latter requires specialized microelectrodes on the chip design and high processing requirements. Also it is too difficult and complex to implement.
Recently, using the conductive solution in microchannel to replace the function of electrode has become a hot. Using the conductive solution in microchannel to replace the function of electrode and the electric field generated by the applied voltage through the ends of the microchannel can emerge the reversible electrical breakdown on the interval between the channels and form electroosmotic flow to drive liquid injector and flow. This can simplify the design process and increase the security. Lee et al. $[13,14]$ designed a liquid electrode microfluidic chip drives by liquid glass electrode for nanofluidics (NLGE). Dielectric breakdown injection microfluidic chip design and experiment are discussed in this paper, which is designed and formed by PDMS material. In order to achieve the dielectric breakdown to drive the electroosmotic flow, we designed a microfluidic channel within the conductive liquid to replace the electrodes to simplify the electrode process of integration, reduce the voltage, and improve the speed of electroosmotic flow. Fluid electrode effect through the dielectric breakdown of the dam between the microchannel could achieve it.

\section{Chip Structure Design}

We simplified the chip structure in order to inject the conductive solution into microchannel conveniently. So, we designed the dam barrier of interlaced microfluidic channel structure (the interval between the two channels was $10 \mu \mathrm{m}$, and 


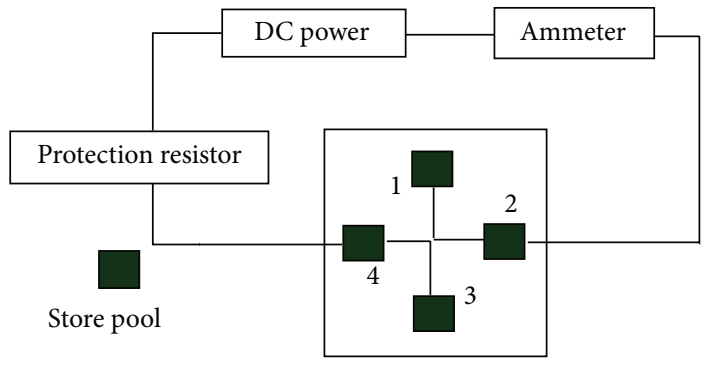

FIgURE 1: The structural and experimental principle chart of electroosmotic flow chip.

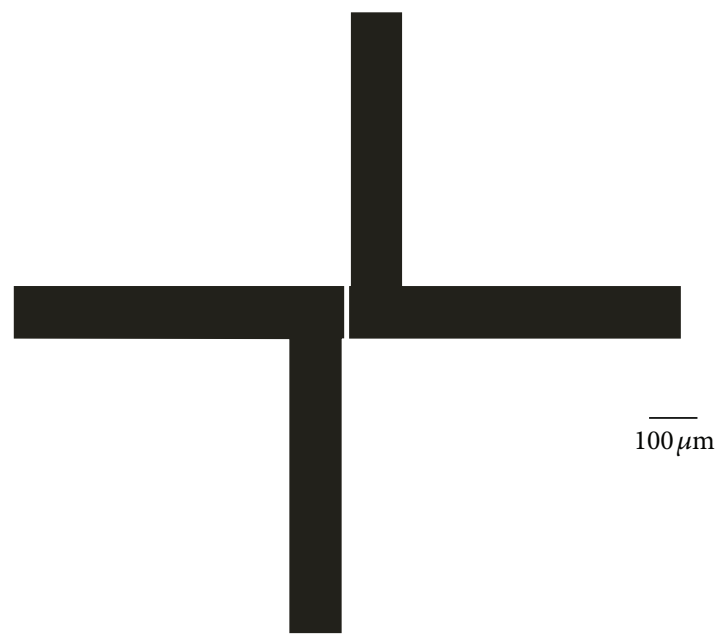

Figure 2: The picture of mask.

the two channels being injected the conductive solution represent the dielectric breakdown electrode). The dielectric breakdown injection microfluidic chip electroosmotic flow driven experiment is shown in Figure 1. The labeled 1, 2, 3, and 4 represent the square sump; the length is $4 \mathrm{~mm}$; the width of the microchannel is about $100 \mu \mathrm{m}$; and the depth is $10 \mu \mathrm{m}$. The distance between the 1,3 or 2,4 sumps is $1 \mathrm{~cm}$.

\section{The Manufacture of Chip}

3.1. The Fabrication of Mask. We draw the mask by Coreldraw software. The front projection of mask version is shown in Figure 2.

3.2. Machining of Chip. Dielectric breakdown microfluidic sample chip is composed of substrate and cover plate bonding, and we all choose the PDMS material in consideration of the needs of the experiment; silicon is selected into substrate's mould made by PDMS.

3.2.1. Mould Making. The mould makes use of lithography and etching processes in silicon in production. The manufacturing steps and process parameters are as follows. Figure 3 is the illustration. Figure 4 is the silicon wafer's lithography graphics.

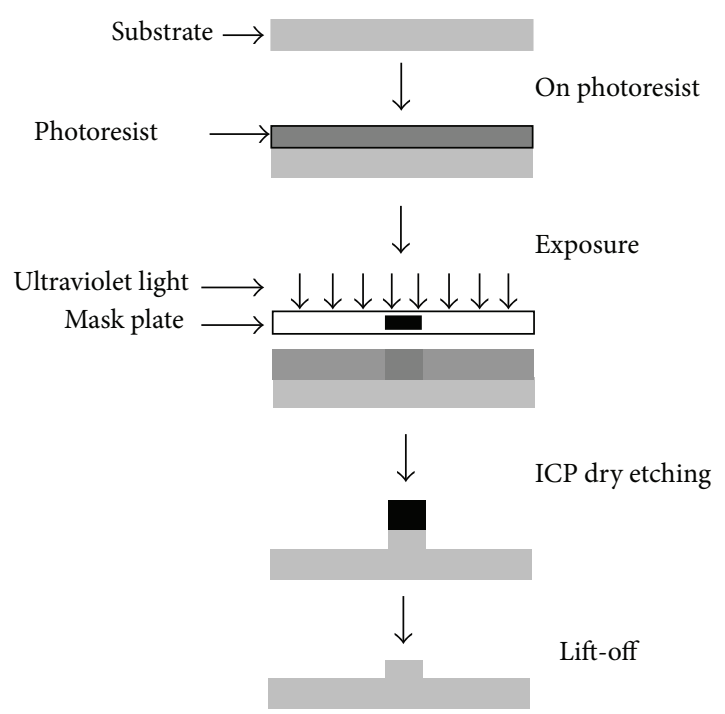

FIGURE 3: Photolithography, the etching process of the silicon wafer.
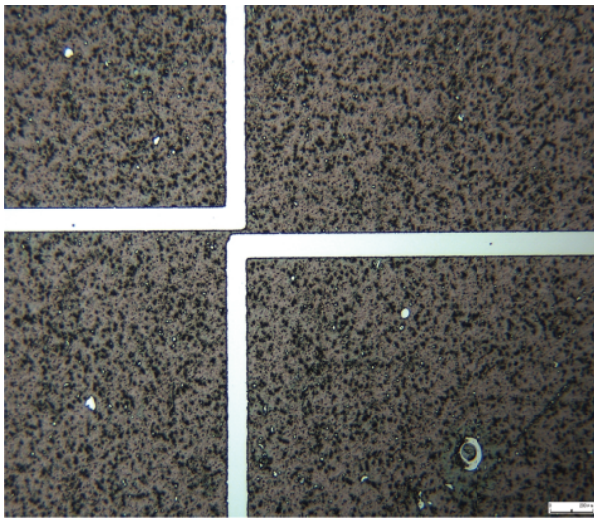

Figure 4: The silicon wafer's lithography graphics photo.

(1) Cleaning: successively use acetone, alcohol, and demonized water ultrasonic cleaning silicon wafers each for 5 minutes, then place it on a hot plate at a temperature of $150^{\circ} \mathrm{C}$ to bake for $1 \sim 3$ minutes after it is dried by nitrogen.

(2) Spin glue: there are two steps to do it. The first step is $500 \mathrm{r} / \mathrm{min}$ rotational $15 \mathrm{~s}$, and second is $4000 \mathrm{r} / \mathrm{min}$ $30 \mathrm{~s}$ rotation.

(3) Preexposure: exposed to ultraviolet for $90 \mathrm{~s}$.

(4) After baking: baked on a hot plate in $112^{\circ} \mathrm{C}$ for $120 \mathrm{~s}$.

(5) Postexposure: exposed to ultraviolet for $160 \mathrm{~s}$.

(6) Displaying picture: development for $40 \sim 45 \mathrm{~s}$ in the liquid.

(7) Inductively coupled plasma (ICP) etching.

Inductively coupled plasma etching method has several superiorities followed as high selectivity, good etching anisotropy, and the ability of getting the plank wall easily; particularly, it has a giant superiority in the deep groove etching 
TABLE 1: Coupling parameters of ICP.

\begin{tabular}{lc}
\hline ICP P/W & 700 \\
RF RP/W & Passivation: 10 \\
& Etching: 25 \\
Gas flow/sccm & Passivation: $\mathrm{C}_{4} \mathrm{~F}_{8}=100$ \\
& Etching: $\mathrm{SF}_{6}=100$ \\
Time/s & Passivation: 10 \\
Cycle number & Etching: 10 \\
Helium pressure/Torr & 30 \\
Total gas pressure/mTorr & 10 \\
Substrate temperature $/{ }^{\circ} \mathrm{C}$ & 40 \\
\hline
\end{tabular}

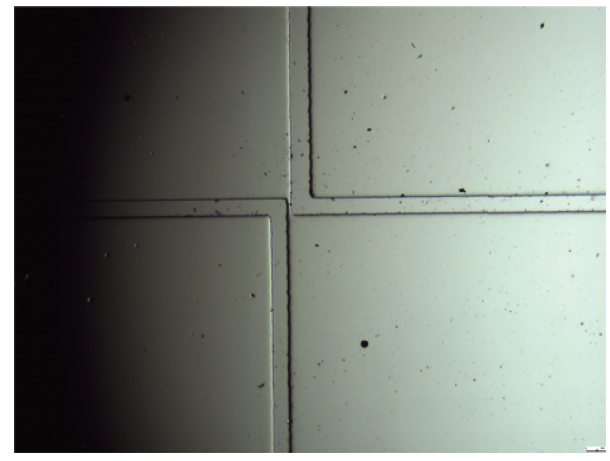

FIGURE 5: Photos with ICP etching on the silicon substrate.

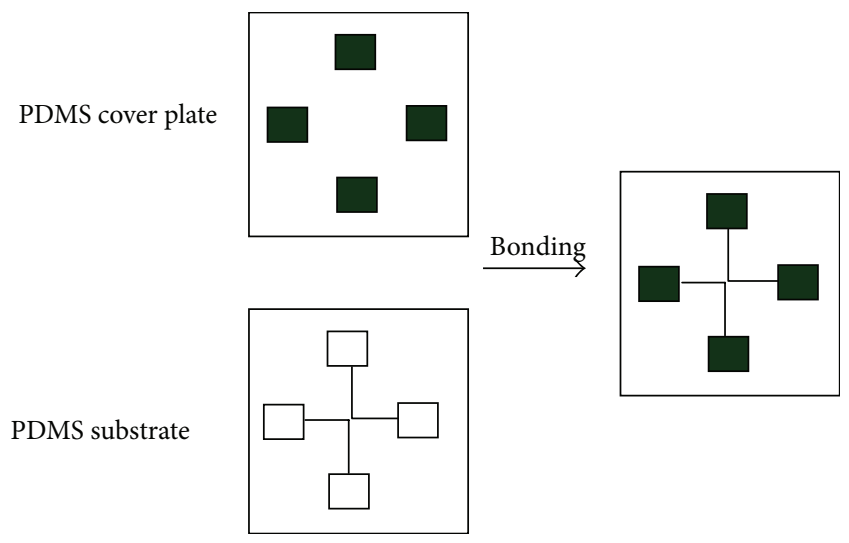

Figure 6: Manufacture process of the chip.

silicon. Inductively coupled plasma ICP etching is one of the technologies about ion.

Etching and its process parameters are showed in Table 1 [15-17]. Figure 5 is the photo of ICP etching.

3.2.2. The Facture of Substrate and Cover Plate. The whole structure and design process of the chip is shown in Figure 6.

(1) PDMS Material Preparation. Firstly, weigh PDMS prepolymer and a curing agent according to the proportion of $10: 1$ by Libra. Secondly, stir for 14 minutes and make them mixed uniformly, then it keep for 30 minutes to eliminate bubbles on

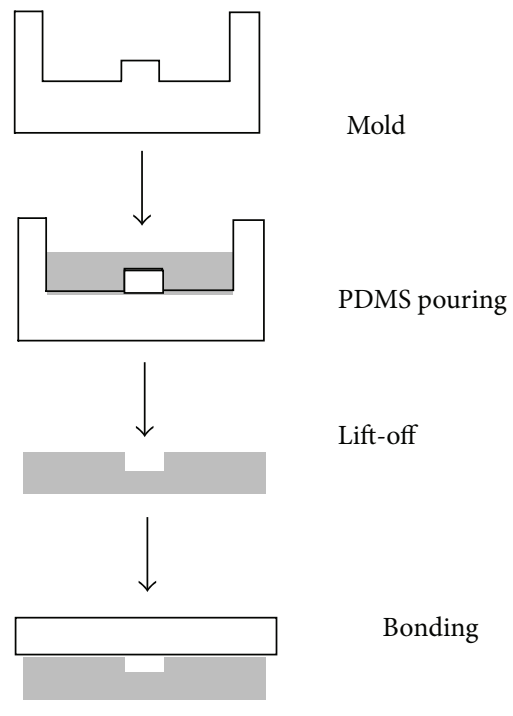

Figure 7: The flowchart of PDMS chip.

the process of stirring, and exhaust it for 1 hour in the vacuum pump to further eliminate bubbles.

(2) Mould Container. Use double-sided adhesive to the surround slide side forming a container, then the silicon is placed in the bottom of the container used as substrate mould. Then, pour the PDMS liquid in the mould, and put the mould at a temperature of $75^{\circ} \mathrm{C}$ for 1 hour [18]. Cut PDMS into the required shape and size with a scalpel.

(3) Making the Cover Plate. Produce the cover plate according to Figure 6. The mould of liquid storage pool is made by rule model with the height $3 \mathrm{~mm}$ and with length and width about $4 \mathrm{~mm}$. The process of making the cover plate is the same as that of substrate. Production flowchart is shown in Figure 7.

3.2.3. The Bonding between the Substrate and the Cover Plate. Place the substrate with a channel structure and the cover plate with the liquid storage pool structure at a distance of $3 \mathrm{~cm}$ away from the $6 \mathrm{~W}$ (the more bigger the power is, the more better the effect is) low pressure mercury lamp $[19,20]$. After exposing for 3 hours, drop the ionized water on cover plate and substrate, then fold it for one minute and place it in the drying box at $65^{\circ} \mathrm{C}$ preserving temperature for 2 hours. Lastly take it and place it for 24 hours to use [18].

\section{Experimental and Discussion of Microfluidic Dielectric Breakdown Sampling Chip}

4.1. Conductive Liquid Filling. Liquid storage pool and microchannel are filled by microsyringe pump whose needle external diameter is $0.9 \mathrm{~mm}$ and internal diameter is $0.46 \mathrm{~mm}$. The picture of operating of microsyringe pump was shown in Figure $8[13,14]$. 


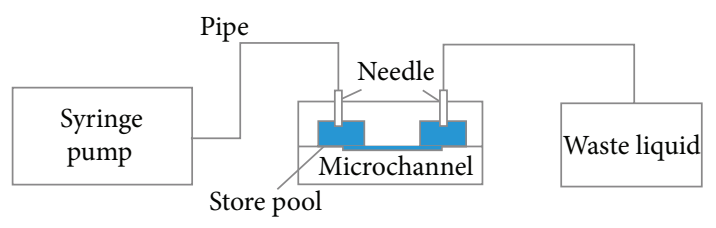

FIGURE 8: The operating system schematic of microsyringe pump.

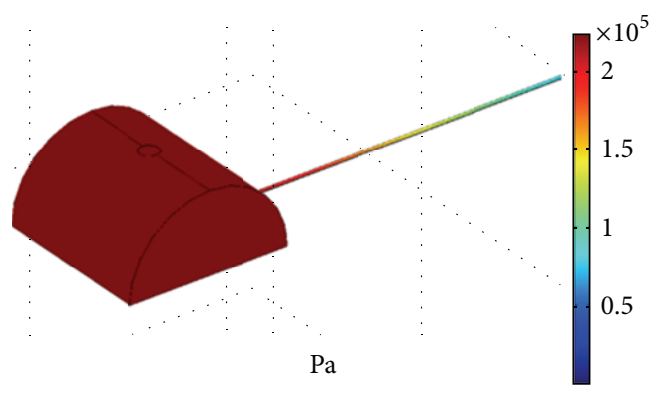

FIGURE 9: Pressure distribution of micropump injection sampling at a speed of $540 \mathrm{uL} / \mathrm{h}$.

The steps of operating the syringe pump are as follows.

(1) Choose the right injector (20,30, and $50 \mathrm{~mL})$, and link the pumped liquid with pipe line. Then, exhaust air, and install the injector on the syringe pump.

(2) Release the press block, fix the injector in a groove, and screw the press block.

(3) Release the push piece, move it to the bottom of injector's pusher, and then fix the pushing piece.

(4) Turn on the switch of the syringe pump, set the pushing speed of the pump as $540 \mathrm{uL} / \mathrm{h}$, open the injection pump, and start sampling. In addition, in order to observe the liquid flow conveniently, the injector should be filled with red ink.

It was found that there is an internal leakage phenomenon of the chip in the sample process, and it is speculated to have these following reasons: one was that the bond was not strong enough and the chip was not fully sealed; another was that the rough surface of the silicon wafer, PDMS, and mould had a little gap through which the liquid could inflow. To solve them, we could test the liquid speed or do its pressure simulation to inspect and confirm the failure of the sample process. The simulation picture was shown in Figure 9.

Through the simulation, we could come to the conclusion that the pressure of microchannel was $26 \times 10^{5} \mathrm{~Pa}$, which was over bonding tolerance pressure of the PDMS $\left(3.45 \times 10^{4} \mathrm{~Pa}\right)$, and we needed to adjust the injection velocity to prevent leakage.

In order to let the pressure be under $3.45 \times 10^{4} \mathrm{~Pa}$, we should adjust the liquid sampling rate. The results (Figure 10) showed that the injection speed rate of $83.7 \mathrm{uL} / \mathrm{h}$ was safe. After resetting the microsyringe injection speed, micropump channel samples successfully and does not leak aging. The color of liquid is red as shown in Figure 11.

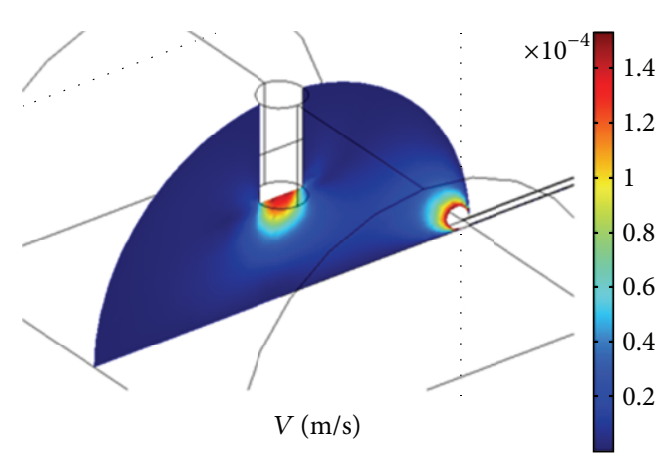

FIGURE 10: The distribution simulation of microinjection pump's speed.

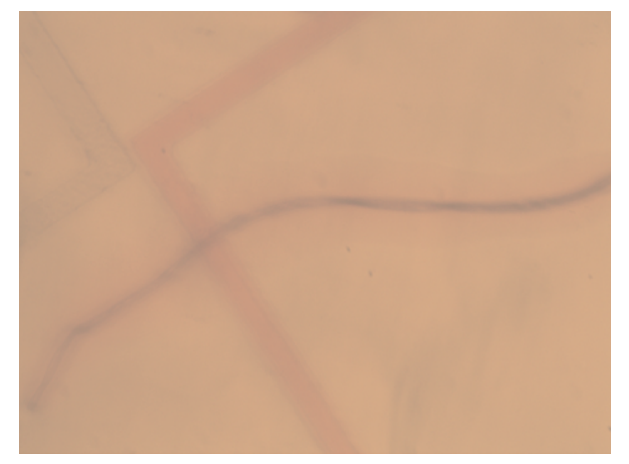

FIGURE 11: The photo under microscope after microchannel samples successfully.

4.2. Dielectric Breakdown Electroosmotic Flow Test. After the success of injecting test liquid, we used DC power to test electroosmotic flow in the chip by measuring the channel current. The schematic diagram of test circuit is shown in Figure 1. When putting the DC voltage on the 2, 4 store pool of the chip, respectively, we could meter the microampere level currents. It could be illustrated that the dam barrier in the chip had dielectric breakdown. Because it had formed a microampere level currents and the current characteristics were consistent with the phenomenon of forming electroosmotic flow it could certify the electroosmotic flow was founded, the flow speed could be determined by the current monitoring method [21]. Thus the project can realize the liquid sampling.

\section{Conclusions}

Using the conductive solution in microchannel to replace the function of electrode and the electric field generated by the applied voltage through the ends of the microchannel can emerge the reversible electrical breakdown on the interval between the channels and form electroosmotic flow to drive liquid injector and flow. This can simplify the design process and increase the security.

We designed dam structure dielectric breakdown chip produced by PDMS to verify the feasibility of dielectric breakdown drive electroosmotic flow in microfluidic chips. 
The experiment proved that using the dielectric breakdown for the liquid sample flow is feasible. This technique can be used to design micropump, microfluidic chip, and so on.

Currently, the theory of the reversibility dielectric breakdown need to a deep research, and the influence on joule heating for reversible breakdown of the chip and the experiment success rate and pressure must be considered. All of these will be worth studying in the future.

\section{References}

[1] G. M. Whitesides, "The origins and the future of microfluidics," Nature, vol. 442, no. 7101, pp. 368-373, 2006.

[2] A. Manz, D. J. Harrison, E. M. J. Verpoorte et al., "Planar chips technology for miniaturization and integration of separation techniques into monitoring systems. Capillary electrophoresis on a chip," Journal of Chromatography, vol. 593, no. 1-2, pp. 253258, 1992.

[3] D. J. Harrison, A. Manz, Z. Fan, H. Lüdi, and H. M. Widmer, "Capillary electrophoresis and sample injection systems integrated on a planar glass chip," Analytical Chemistry, vol. 64, no. 17, pp. 1926-1932, 1992.

[4] D. J. Harrison, K. Fluri, K. Seiler, Z. Fan, C. S. Effenhauser, and A. Manz, "Micromachining a miniaturized capillary electrophoresis-based chemical analysis system on a chip," Science, vol. 261, no. 5123, pp. 895-897, 1993.

[5] R. B. M. Schasfoort, S. Schlautmann, J. Hendrikse, and A. van den Berg, "Field-effect row control for microfabricated fluidic networks," Science, vol. 286, no. 5441, pp. 942-945, 1999.

[6] F. Bianchi, R. Ferrigno, and H. H. Girault, "Finite element simulation of an electroosmotic-driven flow division at a Tjunction of microscale dimensions," Analytical Chemistry, vol. 72, no. 9, pp. 1987-1993, 2000.

[7] L. E. Locascio, C. E. Perso, and C. S. Lee, "Measurement of electroosmotic flow in plastic imprinted microfluid devices and the effect of protein adsorption on flow rate," Journal of Chromatography A, vol. 857, no. 1-2, pp. 275-284, 1999.

[8] J. L. Pittman, C. S. Henry, and S. D. Gilman, "Experimental studies of electroosmotic flow dynamics in microfabricated devices during current monitoring experiments," Analytical Chemistry, vol. 75, no. 3, pp. 361-370, 2003.

[9] Y. Sun, C. S. Lim, A. Q. Liu, T. C. Ayi, and P. H. Yap, "Design, simulation and experiment of electroosmotic microfluidic chip for cell sorting," Sensors and Actuators A, vol. 133, no. 2, pp. 340348, 2007.

[10] Y. C. Lin and W. D. Wu, "Arrayed-electrode design for moving electric field driven capillary electrophoresis chips," Sensors and Actuators B, vol. 73, no. 1, pp. 54-62, 2001.

[11] Y. C. Lin, "Design of low voltage-driven capillary electrophoresis chips using moving electrical fields," Sensors and Actuators $B$, vol. 80, no. 1, pp. 33-40, 2001.

[12] L. M. Fu and R. J. Yang, "Low-voltage driven control in electrophoresis microchips by traveling electric field," Electrophoresis, vol. 24, no. 7-8, pp. 1253-1260, 2003.

[13] S. Lee, R. An, and A. J. Hunt, "Liquid glass electrodes for nanofluidics," Nature Nanotechnology, vol. 5, no. 6, pp. 412-416, 2010.

[14] S. Lee, R. An, and A. J. Hunt, "Supplementary information for Liquid glass electrodes for nanofluidics," Nature Nanotechnology, vol. 10, p. 1038, 2010.

[15] B. Volland, F. Shi, P. Hudek, H. Heerlein, Rangelow, and W. Ivo, "Dry etching with gas chopping without rippled sidewalls,"
Journal of Vacuum Science and Technology B, vol. 17, no. 6, pp. 2768-2771, 1999.

[16] J. H. Min, J. K. Lee, S. H. Moon, and C. Kim, "Deep etching of silicon with smooth sidewalls by an improved gas-chopping process using a Faraday cage and a high bias voltage," Journal of Vacuum Science and Technology B, vol. 23, no. 4, pp. 1405-1411, 2005.

[17] G. D. Boyd, L. A. Coldren, and F. G. Storz, "Directional reactive ion etching at oblique angles," Applied Physics Letters, vol. 36, no. 7, pp. 583-585, 1980.

[18] X. Y. You, D. J. Huang, and Q. Jing, "Study on bonding technique for PDMS microfluidic chips," Analytical Science, vol. 23, pp. 502-506, 2007.

[19] D. C. Duffy, J. C. McDonald, O. J. A. Schueller, and G. M. Whitesides, "Rapid prototyping of microfluidic systems in poly (dimethylsiloxane)," Analytical Chemistry, vol. 70, no. 23, pp. 4974-4984, 1998.

[20] M. Morra, E. Occhiello, R. Marola, F. Garbassi, P. Humphrey, and D. Johnson, "On the aging of oxygen plasma-treated polydimethylsiloxane surfaces," Journal of Colloid And Interface Science, vol. 137, no. 1, pp. 11-24, 1990.

[21] X. Huang, M. J. Gordon, and R. N. Zare, "Current-monitoring method for measuring the electroosmotic flow rate in capillary zone electrophoresis," Analytical Chemistry, vol. 60, no. 17, pp. 1837-1838, 1988. 

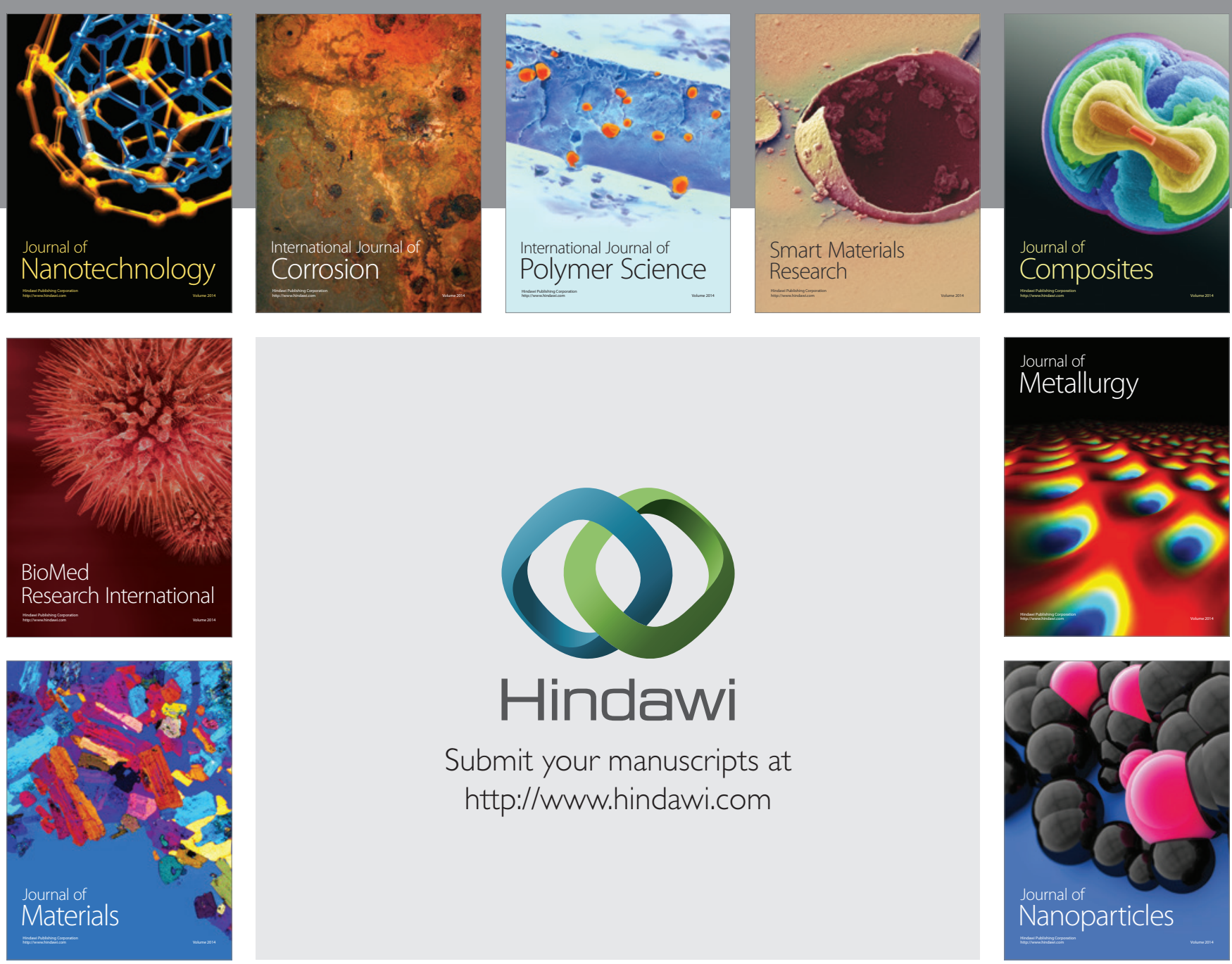

Submit your manuscripts at http://www.hindawi.com
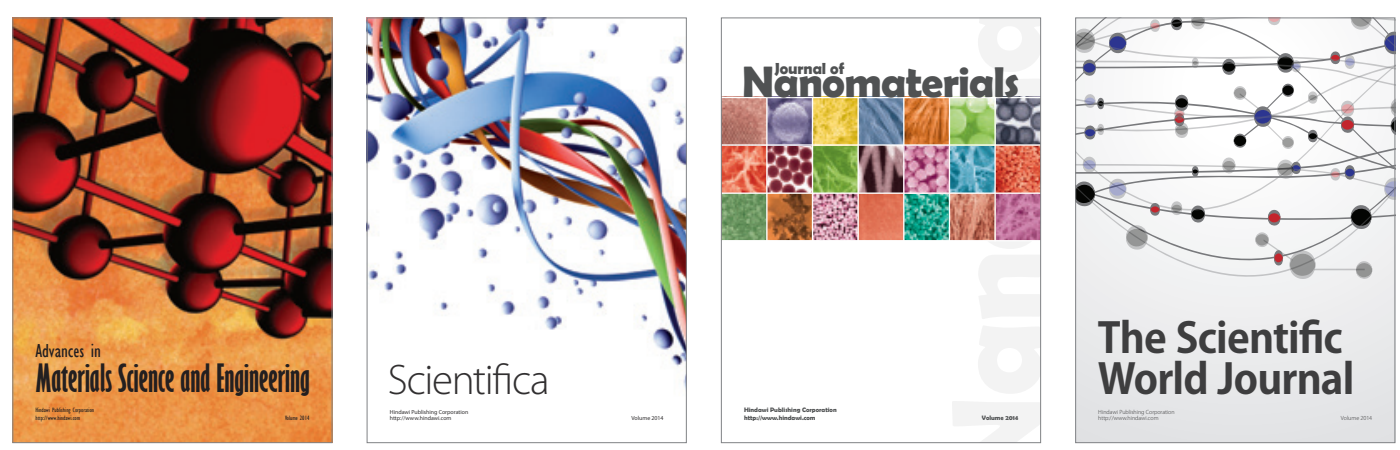

\section{The Scientific World Journal}
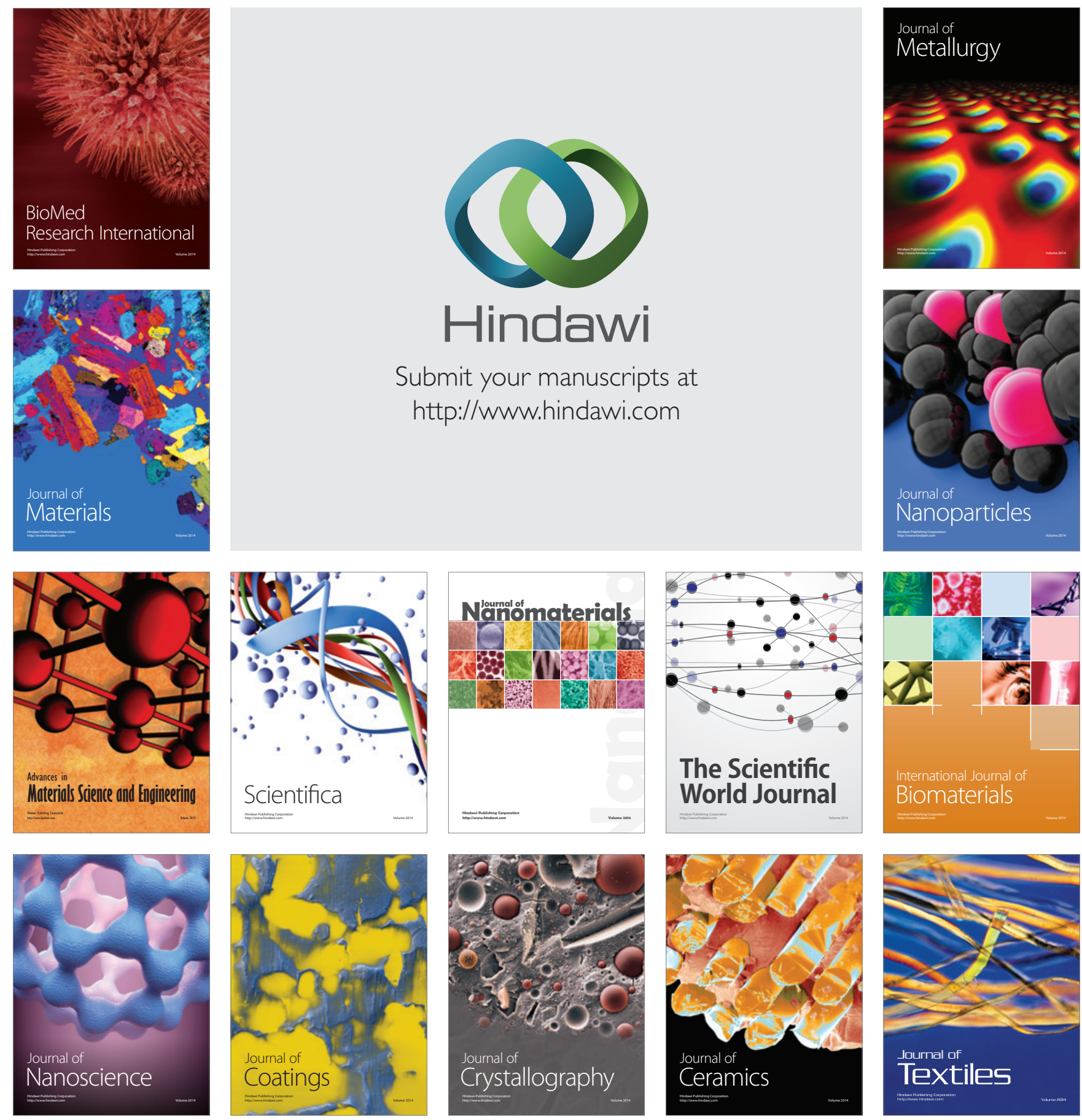